\title{
Primary squamous cell carcinoma of the thyroid: a rare type of thyroid cancer
}

\author{
Pinar Sisman ${ }^{1}$, Ozlem Saraydaroglu ${ }^{2}$, Ahmet Bilgehan Sahin ${ }^{3}$, Ozen Oz Gul ${ }^{4}$, Soner Cander ${ }^{4}$, Canan \\ Ersoy $^{4}$
}

${ }^{I}$ Department of Endocrinology and Metabolism, Kars Harakani State Hospital, Kars, Turkey

${ }^{2}$ Department of Pathology, Uludag University School of Medicine, Bursa, Turkey

${ }^{3}$ Department of Internal Medicine, Uludag University School of Medicine, Bursa, Turkey

${ }^{4}$ Department of Endocrinology and Metabolism, Uludag University School of Medicine, Bursa, Turkey

\begin{abstract}
Primary squamous cell carcinoma of the thyroid is rare but aggressive malignancy. Primary treatment is complete surgical resection of the tumor. If surgical eradication of the tumor is not successful, it has poor prognosis although multimodality treatment. We report a case with multiple thyroid nodules of which fine needle aspiration cytology was suspicious for malignancy. After total thyroidectomy, well differentiated squamous cell carcinoma was observed in histopathological examination. Postoperative investigation ruled out metastasis of the other sites and recurrence has not been observed for 25 months. Early and accurate diagnosis is crucial to achieve complete cure with surgery and long-term survival.
\end{abstract}

Eur Res J 2017;3(1):94-98

Keywords: Squamous cell carcinoma, thyroid, cancer

\section{Introduction}

Primary squamous cell carcinoma (PSCC) of thyroid is a rare type of thyroid malignancy because thyroid gland lacks squamous epithelium. It is seen in less than $1 \%$ among all thyroid malignancies. To our knowledge, during the last 30 years, only about 90 cases were published [1]. The median age is fifth and sixth decade, but PSCC can be seen at any age. It manifests as enlarging mass, pain, and hoarseness. The diagnosis of PSCC should include clinical, endoscopic, radiological, and histological evidence.
Metastatic squamous cell carcinoma (SCC) should always be excluded. In the cases in which direct extension from SCC of adjacent structures to thyroid is observed, exact diagnosis may be difficult. As well as clinical characteristics, optimal treatment strategy of PSCC is poorly defined because of its rarity. PSCC is an aggressive tumor and has poor prognosis although multimodality treatment including surgery, radiotherapy and chemotherapy. It generally has a fatal course within one year of initial diagnosis. 


\section{Case Presentation}

A 60-year-old female patient was admitted to our outpatient clinic for multiple nodules. She had no complaint of dyspnea, dysphagia, hoarseness, weight loss or neck mass. She was asked for malignant risk factors such as radiation, hereditary conditions, thyroiditis, diet, smoking but her past medical and family histories were unremarkable. Her serum free thyroxine (fT4), free triiodothyronine (fT3) and thyroid-stimulating hormone (TSH) levels were 0.9 $\mathrm{ng} / \mathrm{dl}, 1.8 \mathrm{pg} / \mathrm{ml}$, and $3.8 \mu \mathrm{iu} / \mathrm{ml}$, respectively. Other laboratory findings were within normal ranges. Ultrasound revealed one $10 \times 7.5 \mathrm{~mm}$ solid nodule in the right lobe of thyroid, and one $7 \times 5.5 \mathrm{~mm}$ solid nodule in the left lobe. These lesions were oval-shaped and had hypoechoic heterogeneous microstructures (Figure $1 \mathrm{a}, \mathrm{b}$ ). There was no mass and palpable lymph nodes in neck examination. From both nodules, we performed fine needle aspiration biopsy and cytology (FNAC). Pathological examination was benign for the right nodule and was suspicious for malignancy for the left one. Total thyroidectomy was planned. Preoperative calcitonin level was within normal range. Total thyroidectomy was carried out with no complication. During surgery, infiltration of adjacent structures was not observed. In the histopathological examination of the specimen, well-differentiated squamous cell carcinoma was revealed. The specimen

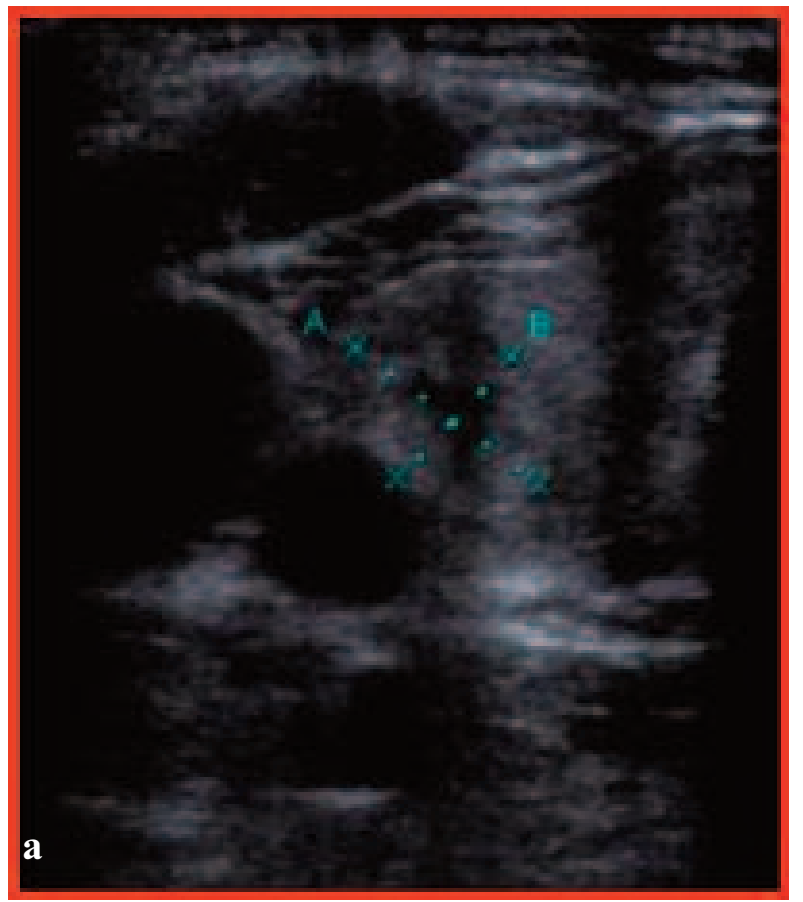

of the right lobe measured $4.5 \times 2.2 \mathrm{~cm}$. There were two benign colloidal nodules in the right lobe. The specimen belonging to the left lobe measured $5.5 \times 4.0$ $\mathrm{cm}$. Tumor was $0.7 \times 0.6 \mathrm{~cm}$ in size. The hematoxylin and eosin (H\&E) stained sections showed invasive, well differentiated squamous cell carcinoma. The neoplastic cells were disposed in sheets, nests and anastomosing cords. Tumoral cells showed marked pleomorphism polygonal-shaped, it consisted of abundant eosinophilic cytoplasm. The tumor had a well-defined capsule formation around. It had extracapsuler extension, but lymphovascular invasion was not observed (Figure 2). By immunohistochemistry, the tumor cells were positive for cytokeratin 5/6. Stains for synaptophysin, chromogranin, and calcitonin were negative. In further immunohistochemical examination, the tumor cells were negative for thyroglobulin and positive for epithelial membrane antigen (EMA), cytokeratin 7 and cytokeratin 19. Ki67 proliferation index was found as increased.

Postoperative examinations of nose, throat and neck by otorhinolaringist and imaging studies (CT and MRI scan of head and neck and F-18fluorodeoxyglucose positron emission tomography) was performed for possible primary malignant lesion of squamous origin causing metastasis to thyroid (Figure 3). Investigations ruled out the possibility of other primary sites. Our case was diagnosed with

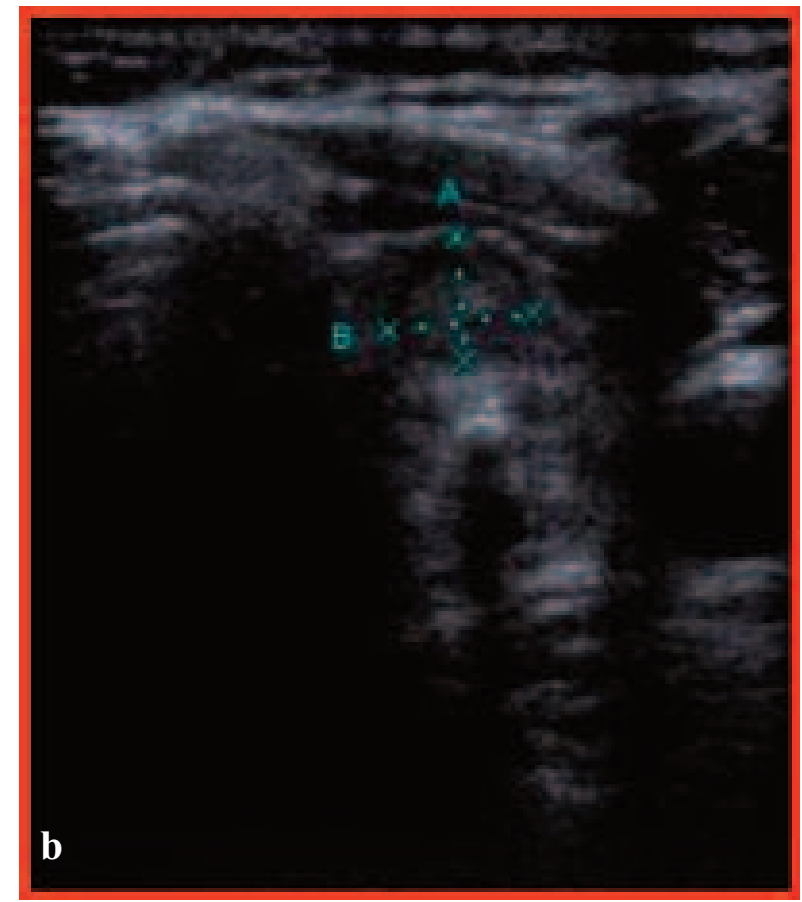

Figure 1. Ultrasound revealed one $10 \times 7.5 \mathrm{~mm}$ solid nodule in the right lobe of thyroid (a), and one $7 \times 5.5 \mathrm{~mm}$ solid nodule in the left lobe (b). These lesions were oval-shaped and had hypoechoic heterogeneous microstructures. 


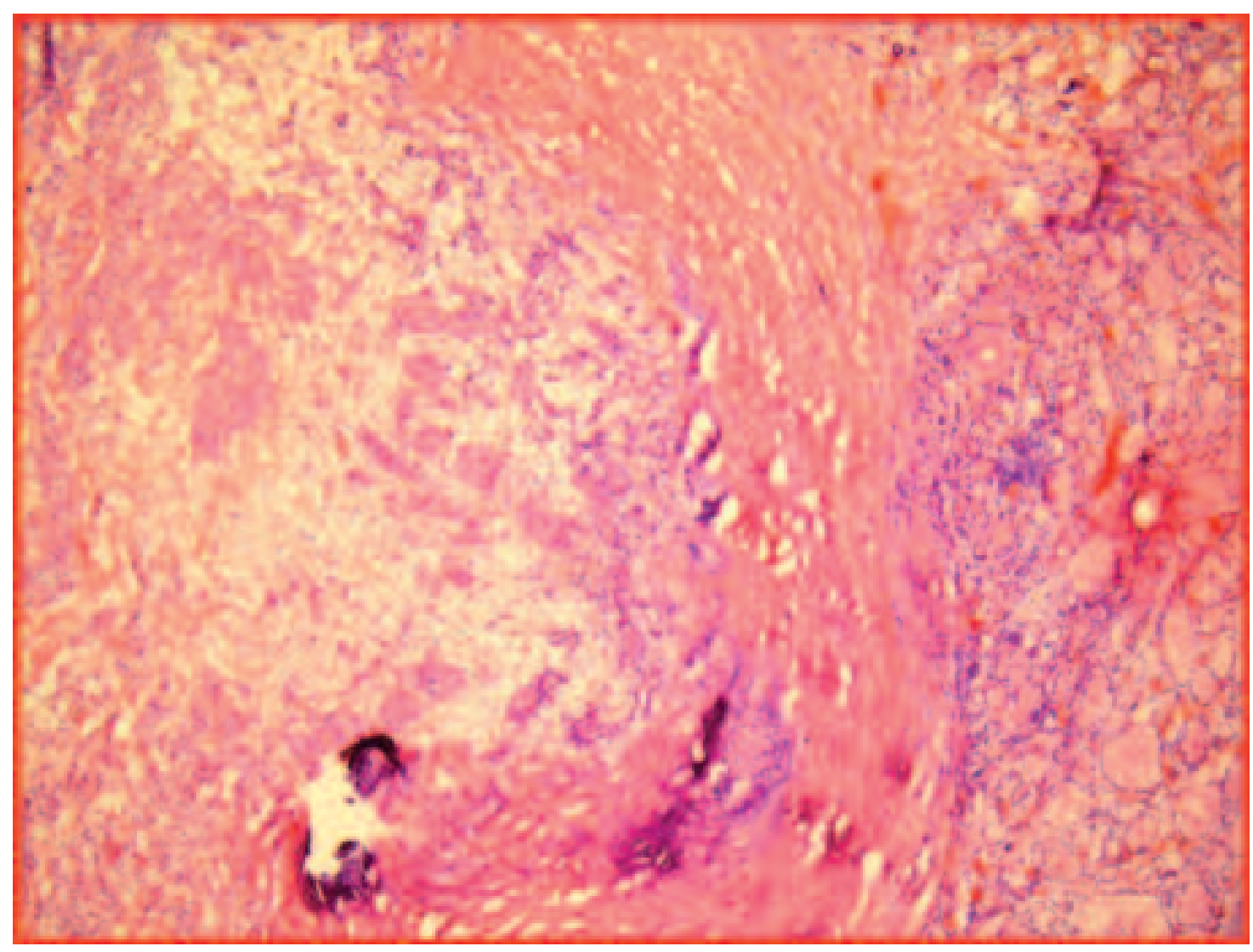

Figure 2. The tumor had a well-defined capsule formation around. It had extracapsuler extension, but lymphovascular invasion was not observed.

PSCC of thyroid by exclusion of metastasis and features of histopathological and immunohistochemical examinations. Because the tumor was locally limited, adjuvant chemoradiotherapy was not considered. Forty-three months have passed after surgery. In CT imaging of neck, chest and abdomen, recurrence has not been observed till now and she has no complaint. She attends her regular outpatient clinic follow-ups.

\section{Discussion}

PSCC of thyroid is a rare malignancy when compared with other thyroid cancers. The reported incidence ranges from $0.7 \%$ to $3.4 \%$ [2-4]. In a systemic review of literature, Cho JK et al. reported that mean age was 63.0 (range; 24-90) and female to male ratio was 2 to 1 [1]. Our patient was a 60 -yearold female in accordance with the literature.

The origin of PSCC of thyroid still remains unclear. There are two theories regarding the pathophysiology of PSCC. The embryonic rest cell theory depends on squamous cell derivation from remnants of the thyroglossal duct and thymic epithelium [5, 6]. According to the second "metaplasia" theory, the source of squamous cells is from follicular, papillary or anaplastic cells [7]. In our patient, Hashimoto's Thyroiditis was not observed in surgical specimen.

Clinical presentation of PSCC of thyroid varies according to tumor's aggressiveness. It may manifest as neck mass, hoarse voice, weight loss, neck pain, dysphagia, dyspnea, stridor [5]. Clinical presentation depends on invasion of tumor to the adjacent structures such as esophagus, trachea etc. Rapidly enlarging neck mass and weight loss should be suggestive for PSCC of the thyroid. The patients may also be asymptomatic as our case, possibly be due to early detection of cancer.

In the diagnosis of PSCC of the thyroid, physical examination, endoscopic, radiological and histopathological studies are crucial. Metastatic SCC of other sites should be excluded. Kidney, lung, head and neck cancers are common sites for thyroid metastasis [8]. So exclusion of metastatic SCC can be provided with endoscopic and radiological imaging. For diagnosis, FNAC is reliable [5]. Kumar et al. [9] 


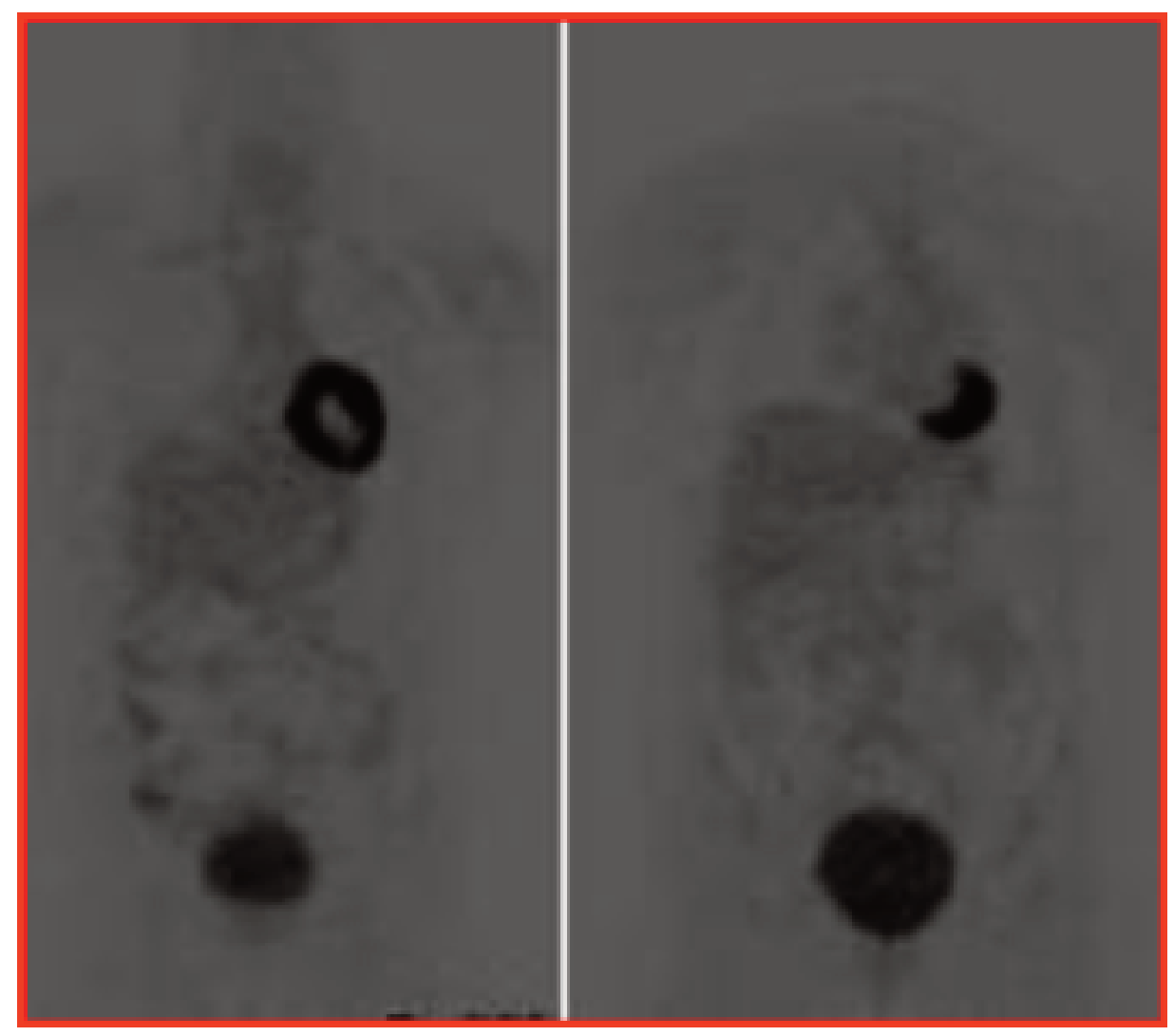

Figure 3. Postoperative positron emission tomography scan was performed for possible primary malignant lesion of squamous origin causing metastasis to the thyroid.

reported that the presence of dyskeratotic, spindle cells and eosinophilic granular keratin material were essential to diagnose SCC in FNAC. Suspicious of high-grade malignancy in cytology combined with clinical, radiological, endoscopic findings can be the most important diagnostic clue for PSCC of the thyroid [10].

Due to rarity of PSCC of the thyroid, treatment modality is not clear. There was no consensus of the extent of surgery, chemotherapeutic agents and radiotherapy. In systemic reviews, treatment with surgery, radiotherapy and chemotherapy alone has been found to be effective and it has extremely poor prognosis $[3,5]$. In the treatment of patients with locally advanced and metastatic disease, combined and aggressive treatments should be considered, but they may not be effective. So, for a long survival, as our patient, early diagnosis and curative surgery with clear margins seem to be crucial.

\section{Conclusion}

In conclusion, PSCC of thyroid is a rare but very aggressive malignancy among thyroid cancers. FNAC is reliable for the diagnosis, but metastatic SCC should be excluded. Complete surgical eradication of tumor is the primary treatment in early stages of the PSCC of thyroid. In locally advanced and metastatic cases, treatment with surgery, radiation therapy and chemotherapy alone has been found ineffective in recent publications. So, early and accurate diagnosis is crucial to achieve complete cure with surgery. For that reason, though rarity, PSCC of thyroid should be kept in mind in patients with thyroid nodules. Evaluation of the specimens by experienced pathologist and excision of the nodules by experienced surgeon are very important in terms of early diagnosis and effective treatment of PSCC of thyroid.

\section{Informed consent}

Written informed consent was obtained from the patient for the publication of this case report.

\section{Conflict of interest}

The authors declared that there are no potential conflicts of interest with respect to the research, authorship, and/or publication of this article. 


\section{References}

[1] Cho JK, Woo SH, Park J, Kim MJ, Jeong HS. Primary squamous cell carcinomas in the thyroid gland: an individual participant data meta-analysis. Cancer Med 2014;3:1396-403.

[2] Heitz P, Moser H, Staub JJ. Thyroid cancer: a study of 573 thyroid tumors and 161 autopsy cases observed over a thirty-year period. Cancer 1976;37:2329-37.

[3] Zimmer PW, Wilson D, Bell N. Primary squamous cell carcinoma of the thyroid gland. Mil Med 2003;168:124-5.

[4] Oz Gul O, Cander S, Sisman P, Erturk E, Ersoy C. Fifteen-year treatment of metastatic thyroid medullary carcinoma: a case report. Eur Res J 2015;1:160-4.

[5] Tunio MA, Al Asiri M, Fagih M, Akasha R. Primary squamous cell carcinoma of thyroid: a case report and review of literature. Head
Neck Oncol 2012;4:8.

[6] Syed MI, Stewart M, Syed S, Dahill S, Adams C, McLellan DR, Clark LJ. Squamous cell carcinoma of the thyroid gland: primary or secondary disease? J Laryngol Otol 2011;125:3-9.

[7] Shrestha M, Sridhara SK, Leo LJ, Coppit GL 3rd, Ehrhardt NM. Primary squamous cell carcinoma of the thyroid gland: a case report and review. Head Neck 2013;35:E299-303.

[8] Hegerova L, Griebeler ML, Reynolds JP, Henry MR, Gharib H. Metastasis to the thyroid gland: report of a large series from the Mayo Clinic. Am J Clin Oncol 2015;38:338-42.

[9] Kumar PV, Malekhusseini SA, Talei AR. Primary squamous cell carcinoma of the thyroid diagnosed by fine needle aspiration cytology. A report of two cases. Acta Cytol 1999;43:659-62.

[10] Lui JT, Khalil MN, Chandarana SP. Primary squamous cell of the thyroid-an abbreviated clinical presentation. J Otolaryngol Head Neck Surg 2014;43:17. 